\title{
NEO-LOGICISM AND RUSSELL'S LOGICISM
}

\author{
Kevin C. Klement \\ Philosophy / U. Mass-Amherst \\ Amherst, MA, OIOO3, USA \\ KLEMENT@PHILOS.UMASS.EDU
}

Certain advocates of the so-called "neo-logicist" movement in the philosophy of mathematics identify themselves as "neo-Fregeans" (e.g., Hale and Wright), presenting an updated and revised version of Frege's form of logicism. Russell's form of logicism is scarcely discussed in this literature and, when it is, often dismissed as not really logicism at all (in light of its assumption of axioms of infinity, reducibility and so on). In this paper I have three aims: firstly, to identify more clearly the primary meta-ontological and methodological differences between Russell's logicism and the more recent forms; secondly, to argue that Russell's form of logicism offers more elegant and satisfactory solutions to a variety of problems that continue to plague the neo-logicist movement (the bad company objection, the embarrassment of richness objection, worries about a bloated ontology, etc.); thirdly, to argue that neo-Russellian forms of logicism remain viable positions for current philosophers of mathematics.

\section{INTRODUCTION}

I $\mathrm{t}$ was for a while fashionable to say that logicism was dead. Even now we see the sentiment repeated. ${ }^{\mathrm{I}}$ As recently as 20IO, a website developed for a popular logic textbook ${ }^{2}$ claimed that "in the 1920's, the German mathematician and logician Kurt Gödel made an astonishing discovery, a discovery that effectively ended the hopes of the logi-

${ }^{\mathrm{I}}$ For other examples, see Scott Soames, Philosophical Analysis in the Twentieth Century, Vol. I: The Dawn of Analysis (Princeton: Princeton U. P., 2003), p. I57, and Arthur Sullivan, ed., Logicism and the Philosophy of Language: Selections from Frege and Russell (Peterborough, Ont.: Broadview P., 2003), p. 85.

${ }^{2}$ See Paul Herrick, website for The Many Worlds of Logic (Fort Worth: Harcourt Brace, 1994), http://www.manyworldsoflogic.com, (C) 2004; accessed January 2010. 
cists", namely, that "there are truths of arithmetic that are in principle not provable", and "with tight deductive proofs that convinced everyone" (!), Gödel proved that "logicism is false", and indeed:

Today, logicians agree that although mathematics can be derived from set theory, it cannot be derived from pure logic alone. Thus was an exciting new theory finally proven false.

Apparently, I need to turn in my logician card and even my someone card, since I'm not aware of Gödel having proven (or even having attempted to prove) any such thing. ${ }^{3}$

However, the tendency to make such bold pronouncements is on the decline. The thanks for this decline are owed largely to the attempts by certain thinkers to resuscitate a form of logicism in recent decades. The most influential attempt is found in the work of Crispin Wright along with his collaborators, principally Bob Hale. ${ }^{4}$ Wright and Hale both frame their position as a kind of neo-Fregeanism. Given the inconsistency of Frege's Grundgesetze, the kind of logicism they advocate cannot be the same as Frege's down to the letter-even Frege himself did not endorse Fregean logicism at the end of his life-but they describe their position as an attempt to salvage Frege's overall philosophy of mathematics. They compare the details of their positions to his, they discuss at length particular puzzles Frege himself belaboured, they endorse principles they attribute to Frege, and Frege's work is constantly portrayed as a source of inspiration. I have some serious doubts as to what extent the view they end up endorsing can really be understood as a form of neoFregeanism (indeed, parts of it are decidedly anti-Fregean), but that is a topic for another occasion.

${ }^{3}$ What is particularly strange about Herrick's way of putting the point is that he somehow thinks Gödel's results pose a greater problem for reducing mathematics to logic than to set theory, whereas of course Gödel's results equally show that no recursively axiomatized set-theoretic foundation for mathematics is complete.

${ }^{4}$ See especially Crispin Wright, Frege's Conception of Numbers as Objects (Aberdeen: Aberdeen U. P., I983), and Bob Hale and Crispin Wright, The Reason's Proper Study: Essays towards a Neo-Fregean Philosophy of Mathematics (Oxford: Clarendon P., 200I). Neil Tennant and Stewart Shapiro have also done work in sympathy with their programme, though without the same kind of commitment to the project. Moreover, George Boolos, Richard Heck and Kit Fine have contributed in important ways, though none of them, I think, actually endorse the position in the end. 
In this paper, I instead hope partly to redress the nearly complete lack of interplay between the work done by Wright-style neo-logicists and the other pillar of historical logicism, Bertrand Russell. It would not be fair to say that Wright ignores Russell altogether; Russell's views on indefinitely extensible concepts are discussed in some detail in Wright and Shapiro's discussions of neo-logicist set theories, for example. ${ }^{5}$ But I think it is fair to say that Wright, and those of his school, almost completely ignore Russell's form of logicism, either as a source of inspiration and guidance for their own, or as a rival approach in the philosophy of mathematics their deviance from which needs justifying. A widespread position seems to be that Russell's form of logicism wasn't really logicism at all, once it is fully understood. Here is what is, as far as I know, the most extended statement Wright makes about Russell's form of logicism:

With one or two exceptions, virtually no one nowadays accepts that there is any sort of truth wrapped up in number-theoretic logicism. Some, maybe, would grant that all depends on what we take to be comprised in "logic". But the general view would seem to be that Russell's Paradox put paid to Frege's attempt, and that Russell's own attempt in Principia Mathematica is vitiated by the manifestly non-logical character of its axiomatic basis (displayed most signally by the Axiom of Infinity) and by the incompleteness theorems of Gödel. But these are bad reasons. ${ }^{6}$

Wright goes on to explain why it is that Gödel's results do not vitiate logicism. His explanation is, as far as I can tell, irreproachable, and ought to be something I needn't rehearse; but quickly for those who may be unfamiliar, what the Gödel results show is that there is no recursively axiomatized system for number theory that is complete, i.e., contains every number-theoretic truth as a theorem. If we understand logicism to be the thesis that mathematical truths are logical truths, Gödel's results would only pose an impediment to logicism if we were somehow convinced that logic itself needed to be capable of a complete recursive axiomatization. If not, all the results show is the impossibility of recursively axiomatizing logic itself. Indeed, second-order logic is known to be

5 See, e.g., Shapiro and Wright, "All Things Indefinitely Extensible", in Absolute Generality, ed. A. Rayo and G. Uzquiano (New York: Oxford U. P., 2007), pp. 255-304.

${ }^{6}$ Wright, Frege's Conception of Numbers as Objects, pp. I3O-I; on this point it is worth comparing Bernard Linsky and Edward Zalta, "What Is Neo-Logicism?", The Bulletin of Symbolic Logic I2 (2006): 60. 
incomplete due to a corollary of Gödel's results. Hence, those results don't establish the falsity of the thesis that mathematical truths are logical truths any more than they establish the falsity of the supposition that second-order logical truths are logical truths. ${ }^{7}$ (Of course, there are those who doubt that second-order logic is really logic, ${ }^{8}$ but if that is a problem for logicism, it is a different problem from Gödel's results.)

Wright does not discuss the other alleged flaw in Russell's logicism, the need for an axiom of infinity, though given Wright's own views, he could not in principle hold that it couldn't be understood as any kind of logical truth. Indeed, Wright does not discuss Russell's logicism further.

In what follows, I want to argue that this is unfortunate, and symptomatic of a general trend among working philosophers of mathematics not to take Russell's logicism as seriously as it should be. This is in part because Russell's views are not well understood. In particular, few seem to understand how Russell's views constitute a form of logicism at all, given the seemingly non-logical principles of infinity, choice and reducibility. For the neo-logicists, and others interested in assessing the contemporary neo-logicist movement, the neglect of Russell's work is all the more unfortunate, precisely because I think Russell gave serious thought to issues, if not precisely the same as, then at least very similar to the most important outstanding puzzles and potential problems that the current neo-logicists face. I argue that Russell's considered views on these matters are more sophisticated than is widely known, and indeed, that Russell came to different answers largely as a result of a different metaontological and methodological starting point. I believe Russell's views have the resources to provide eloquent and satisfactory solutions to the difficulties faced by contemporary logicists, though they do not come without a cost. Taking a Russellian approach also means abandoning the

7 For a more sophisticated look at the importance of Gödel's results in assessing logicism, see Agustín Rayo, "Logicism Reconsidered", in The Oxford Handbook of the Philosophy of Mathematics and Logic, ed. Shapiro (Oxford: Oxford U. P., 2005). Rayo there argues that the Gödel results primarily pose a problem for proof-theoretic logicisms that hold that mathematical truths can be deduced in a single recursively axiomatized logical system. Disappointingly, however, Rayo does not discuss proof-theoretic forms of logicism that hold only the weaker view that for every mathematical truth, there is some recursively axiomatized system or other in which it can be proven, which, as far as I can tell, is untouched by the Gödel theorems.

${ }^{8}$ See, e.g., W. V. Quine, The Philosophy of Logic, 2nd edn. (Cambridge, Mass.: Harvard U. P., 1986), and John Burgess, Fixing Frege (Princeton: Princeton U. P., 2005). 
rather more simple but perhaps naïve connection between language and ontology endorsed by Wright et al. In the end, I think the prospects for neo-Russellian neo-logicisms are, if anything, more promising than the currently dominant alternatives.

\section{CONTEMPORARY ABSTRACTIONIST NEO-LOGICISM}

I begin by sketching Wright- and Hale-style neo-logicism, as well as the chief difficulties it faces. It should be noted that there have been other, sometimes very different, attempts by other scholars to justify some form of logicism or another in recent years. These include such thinkers as Bostock, Cocchiarella, Landini, Sher, Hodes, Linsky and Zalta, to name a few, ${ }^{9}$ some of whom take better account of Russell's work, but unfortunately I shall not be able to give these rival neo-logicisms the attention they deserve. My focus will be on those forms of neo-logicism that give a primary role to abstraction principles, or "Abstractionism", as it is occasionally called.

Wright usually stops short of saying that arithmetical truths are logical truths or that arithmetic is simply a species of logic; he prefers to say that arithmetic can be shown to be analytically true, and hence has an epistemological and metaphysical status similar to that afforded to it by Frege. Arithmetical truths are entailed by the axioms of second-order logic supplemented with certain additional principles that are themselves analytical truths or have the epistemological and metaphysical status of definitions. These additional principles paradigmatically take the form of abstraction principles. These are principles taking the following form:

$$
\forall \kappa \forall \tau(\Sigma(\kappa)=\Sigma(\tau) \leftrightarrow E(\kappa, \tau))
$$

Here, " $\kappa$ " and " $\tau$ " are two variables of the same logical type (e.g., two

${ }^{9}$ See David Bostock, Logic and Arithmetic: Natural Numbers (Oxford: Clarendon P., 1974); Nino Cocchiarella, "Frege, Russell and Logicism: a Logical Reconstruction", in Logical Studies in Early Analytic Philosophy (Columbus: Ohio State U. P., 1987); Gregory Landini, "Russell's Intensional Logic of Propositions: a Resurrection of Logicism?", in Thought, Language and Ontology, ed. F. Orilia and W. J. Rappaport (Amsterdam: Kluwer, 1998); Gila Sher, "A Conception of Tarskian Logic”, Pacific Philosophical Quarterly 70 (1989): 34I-68; Harold Hodes, "Logicism and the Ontological Commitments of Arithmetic", Journal of Philosophy 8I (1984): 123-49, and Linsky and Zalta, "What is Logicism?". 
individual variables, two monadic predicate variables, two dyadic relation variables, etc.), " $\Sigma$ " is a function sign operating on expressions of the type of $\kappa$ and $\tau$ and forming an individual term, and " $E$ " stands for some equivalence relation between things of the type of $\kappa$ and $\tau$. Such abstraction principles, when legitimate, are understood by Wright and Hale as implicitly defining the function sign " $\Sigma$ " and, by means of stipulating their identity conditions, introducing the entities denoted by terms of the form " $\Sigma(\kappa)$ " into the domain of discourse.

The most important such principle for number theory is what has come to be called Hume's principle, ${ }^{\mathrm{IO}}$ which can be stated as follows:

$$
\forall F \forall G(\#(F)=\#(G) \leftrightarrow F \cong G)
$$

where " $F \cong G$ " abbreviates the equivalence relation of equinumerosity, definable using second-order logical constants alone, that holds between $F$ and $G$ when the $F$ s and the $G$ s can be put in I-I correspondence with one another.

$$
\begin{aligned}
F \cong G={ }_{\mathrm{df}} \exists R(\forall x[F x \rightarrow & \exists y(G y \wedge R x y)] \wedge \forall y[G y \rightarrow \exists x(F x \wedge R x y)] \wedge \\
& \forall x \forall y \forall z[(R x y \wedge R x z) \vee(R y x \wedge R z x) \rightarrow y=z])
\end{aligned}
$$

Read together with this definition, Hume's principle can be read as saying that the number of $F$ s is the number of $G$ s if and only if $F$ and $G$ are equinumerous. Hume's principle, together with the standard axioms and rules for second-order logic, suffices for the proof of second-order Peano arithmetic. Other abstraction principles are thought sufficient to establish the basic truths of the real numbers and even set theory. ${ }^{\text {II }}$

Obtaining the Peano postulates from Hume's principle is perhaps the most important case for the evaluation of Abstractionist neo-logicism, and it is worth pausing briefly to discuss how the result is obtained. The

${ }^{\text {Io }}$ For perhaps not very good reasons; see Michael Dummett, "Neo-Fregeans: in Bad Company", in The Philosophy of Mathematics Today, ed. Matthias Schirn (Oxford: Clarendon P., I998), pp. 386-7.

II See Hale, "Reals by Abstraction", in Reason's Proper Study, pp. 399-420; George Boolos, "Saving Frege from Contradiction", in Logic, Logic and Logic (Cambridge, Mass: Harvard U. P., I998), pp. I7I-82; Shapiro, "Prolegomenon to Any Future Neo-Logicist Set Theory", British Journal for the Philosophy of Science 54 (2003): 59-9I; Shapiro and Wright, "All Things Indefinitely Extensible". 
Peano postulates may be stated using the notion of zero, the successor relation, and the notion of a natural number. These may be defined using the \#( ) functor introduced by Hume's principle as follows:

$$
\begin{aligned}
& 0={ }_{\mathrm{df}} \#(\lambda x(x \neq x)) \\
& S x y={ }_{\mathrm{df}} \exists F \exists z[F z \wedge y=\#(F) \wedge x=\#(\lambda w(F w \wedge w \neq z))] \\
& N x={ }_{\mathrm{df}} \forall F[F(0) \wedge \forall y \forall z(F y \wedge S y z \rightarrow F z) \rightarrow F x]
\end{aligned}
$$

The Peano postulates are then:

$$
\begin{aligned}
& \text { I. } N(0) \\
& \text { 2. } \forall x[N x \rightarrow \exists y \forall z(N z \wedge P x z \leftrightarrow y=z)] \\
& \text { 3. } \forall x \neg S(x, 0) \\
& \text { 4. } \forall x \forall y \forall z(S y x \wedge S z x \rightarrow y=z) \\
& \text { 5. } \forall F[F(0) \wedge \forall y \forall z(F y \wedge S y z \rightarrow F z) \rightarrow \forall x(N x \rightarrow F x)]
\end{aligned}
$$

The most difficult, by far, of these to obtain is the second, which states that every natural number has a unique successor that is a natural number. The uniqueness part is not hard to establish, the existence part is. It requires showing that for every natural number $n$, there is a concept that is instantiated by $n$ many things, plus one more. The proof of this from Hume's principle requires making use of the numbers themselves. Inductively, we have to establish that there is a concept instantiated by one more than zero-many things, namely the concept of being identical to zero. This establishes the existence of one. Once one is on the table, we can use this to establish the existence of two, since the concept of being a natural number less than or equal to one is instantiated by two things. The concept of being a natural number less than or equal to two is then instantiated by three things, and so on. ${ }^{\text {I2 }}$

This testifies to the power of Hume's principle; from it alone, one can establish the existence of infinitely many numbers, which of course is necessary, since the Peano postulates cannot be satisfied within a finite domain. For later discussion, I shall refer to the phenomenon as "bootstrapping"; one concept, guaranteed by logic to be instantiated by nothing, can be used with Hume's principle to establish the existence of one

${ }^{12}$ For more of the proof of the Peano postulates in the second-order logical calculus plus Hume's principle, see Richard Heck, “The Development of Arithmetic in Frege's Grundgesetze der Arithmetik", Journal of Symbolic Logic 58 (1993): 579-600. 
number. Once we have it, we can establish another, and so on, ad infinitum. This would not be possible if Hume's principle were not formulated so that the entities named by the terms flanking the identity sign on the left could not be instances of the concepts $F$ and $G$, or values of the quantified individual variables that occur in the full expression of the right side.

All the more so given its deductive power, Wright owes us an explanation for the claim that Hume's principle can be thought of as an analytic truth, as having, roughly, both the epistemological and metaphysical status of a definition. It is not a standard sort of definition; it does not allow us to eliminate the sign "\#( )" from all those contexts in which it appears. Nevertheless, Wright believes that Hume's principle is an analytic consequence of the notion of number, that it, in effect, explains what we mean when we speak of a number, and that anyone who properly understands the formula of second-order logic that occurs on the right side of it and is in a position to recognize an instance of it as true or false is in a position to become aware of the existence of one or (in the case of a false instance) two numbers. Borrowing a metaphor from Frege, ${ }^{13}$ Wright describes an instance of the left side as a "recarving" or reconceptualization of the content of the corresponding instance on the right. I have never found this metaphor particularly helpful. It seems to suggest that the thoughts expressed by certain formulae can be analyzed as having a structure, but fluidly, so that the same thought is capable of radically disparate decompositions depending on the precise form of articulation. It is highly unlikely that Frege's own views on the nature of thoughts is compatible with such a possibility. ${ }^{\text {I4 }}$ But the issue here is not Frege's views, but Wright's, and thankfully he does have more to say about it. Wright endorses the view, which he also attributes (problematically, I think) to Frege, that truth is constitutively prior to reference. What it is for language to refer to objects is for certain expressions which have the syntactic and combinatorial properties of singular terms to serve as meaningful and discrete parts of appropriate kinds of true sentences.

${ }^{13}$ Gottlob Frege, Die Grundlagen der Arithmetik, translated as The Foundations of Arithmetic (Evanston: Northwestern U. P., 1974), \$64.

${ }^{\mathrm{I}} 4$ As I have argued elsewhere; see Klement, Frege and the Logic of Sense and Reference (New York: Routledge, 2002), Ch. 3, and "Grundgesetze and the Sense/Reference Distinction”, in A Companion to Frege's Grundgesetze der Arithmetik, ed. P. Ebert and M. Rossberg (Oxford: Oxford U. P., forthcoming). 
... when it has been established, by the sort of syntactic criteria sketched, that a given class of terms are functioning as singular terms, and when it has been verified that certain appropriate sentences containing them are, by ordinary criteria, true, then it follows that those terms do genuinely refer. And being genuine terms, their reference will be to objects. There is to be no further, intelligible question whether such terms really have a reference, whether there really are such objects. ${ }^{15}$

We are free, then, to introduce terms such as those formed with “\#( )”, and provided we are able to fix the truth conditions of statements in which they appear, and fix their identity conditions-we may do so stipulatively_and can recognize independently the obtaining of these conditions, this is enough to guarantee that these terms refer. This is precisely what Hume's principle does with regard to the sortal concept of number; it fixes the identity conditions of numbers in terms of an already understood logical vocabulary, and thereby fixes the truth conditions of arithmetical statements in which statements about numbers appear.

\section{PROBLEMS WITH ABSTRACTIONIST NEO-LOGICISM}

It is difficult to deny the intuitive appeal of Wright's position. Hume's principle does seem in some way an explanation of what we mean when we speak of numbers; if it does not have the status of a kind of definition or other kind of analytical truth, it is difficult to understand what other sort of status it could have, on what other ground we could come to know it or even on what other ground it could be true. The problems are in part technical, in part meta-ontological. I shall limit my discussion to certain issues, those most germane to my discussion of Russell's work to follow.

\section{I The Bad Company Objection}

Perhaps the most notorious problem is known as the Bad Company Objection. ${ }^{16}$ Nothing in what we've seen so far in Wright's defence of the analyticity of Hume's principle suggests that there would be anything

Is Wright, Frege's Conception of Numbers as Objects, p. I4.

${ }^{16}$ See Dummett, "Neo-Fregeans: in Bad Company", and Boolos, "Is Hume's Principle Analytic?", in Logic, Logic and Logic, pp. 30I-I4. 
wrong with taking any abstraction principle of form (AP) as a kind of stipulative definition. But is it true that starting from any equivalence relation, we can abstract entities, one for each group of equivalents-any equivalence relation at all? The answer is no. There are abstraction principles or implicit definitions of almost precisely the same form as Hume's principle which, far from being analytical truths, are demonstrably logically impossible. The most famous is Frege's Basic Law V, which Frege himself blamed for the derivability of Russell's paradox within the inconsistent system of his Grundgesetze. Reconstructed within a modern second-order predicate calculus, it is typically stated as follows:

$$
\forall F \forall G(\operatorname{Ext}(F)=\operatorname{Ext}(G) \leftrightarrow \forall x(F x \leftrightarrow G x))
$$

Concepts $F$ and $G$ have the same extension if and only if they are coextensional. It would not be difficult to imagine someone ignorant of the resulting contradiction taking this as not only analytically true, but stipulatively true, true in virtue of a decision about what is to be meant by "the extension of a concept". Yet such a person would be guilty of some kind of logical mistake. Wright owes us an explanation of the difference.

And Basic Law V is not the only problematic example. One rarely discussed case involves what you might take to be the equivalence relation par excellence, identity. ${ }^{17}$

$$
\text { (Things) } \quad \forall F \forall G(\operatorname{Thing}(F)=\operatorname{Thing}(G) \leftrightarrow F=G)
$$

But this just amounts to the supposition that a unique object exists for every concept, a clear violation of Cantor's theorem, and leads immediately to Russell's paradox of predication.

Another "bad" abstraction principle which should be particularly worrisome for the Abstractionist neo-logicist, given its great apparent similarity to (HP) is this:

$$
\forall R \forall S(\operatorname{Ord}(R)=\operatorname{Ord}(S) \leftrightarrow R \approx S)
$$

Here " $R \approx S$ " abbreviates the formula (again expressible using purely

${ }^{17}$ Those who demur from identity between concepts may utilize indiscernibility here instead, i.e., $\forall M(M(F) \leftrightarrow M(G))$, or more Fregeanly, $\forall M\left(M_{\beta}(F(\beta)) \leftrightarrow M_{\beta}(G(\beta))\right)$. 
logical vocabulary) claiming that $R$ and $S$ are isomorphic or ordinally similar relations. The abstraction says the order-type of relation $R$ is the order-type of relation $S$ if and only if $R$ and $S$ are isomorphic. This principle yields a contradiction from the Burali-Forti paradox. ${ }^{18}$ Yet, it seems to be nothing more nor less than Hume's Principle modified to deal with ordinals rather than cardinals. ${ }^{19}$ Of course, there are some differences between this and (HP), most notably (HP) does seem to be consistent (and indeed is, if second-order Peano arithmetic is). But this does not explain how it can serve the sort of foundational role Wright wants it to play, given the bad company it keeps. Indeed, we have very good reason to think that consistency alone cannot salvage any privileged status for abstraction principles, but this leads into the next problem.

\subsection{The Embarrassment of Riches Objection}

Not only are there abstraction principles, which, taken in isolation, lead to contradiction, there are pairs of abstraction principles, each of which is consistent, but incompatible with one another. ${ }^{20}$ We need not look at too many examples; it'll suffice in this context to examine one. Hume's principle, in leading to full second-order Peano arithmetic, is only satisfiable in infinite domains. There are other consistent abstraction principles that are only satisfiable in finite domains, one of which is Boolos's parity principle, stated as follows:

$$
\forall F \forall G(\operatorname{Parity}(F)=\operatorname{Parity}(G) \leftrightarrow F \ominus G)
$$

Where " $\ominus$ " represents the (equivalence) relation of differing evenly that holds between $F$ and $G$ iff there is an even number of objects falling under one but not the other.

With these sorts of examples, the neo-logicist cannot cite the inconsistency of the abstraction principle as grounds for rejecting it, since (PP) is consistent on its own. In conjunction with (HP), however, we get a contradiction. Wright et al. must give some non-ad hoc explanation for why it is (PP), not (HP), that we should reject, and why one must be

${ }^{18}$ See Hodes, "Logicism and the Ontological Commitments of Arithmetic", p. I38n.

${ }^{19}$ For the connection between order-types and ordinals, see IMP, Ch. 7.

${ }^{20}$ See Boolos, "The Standard Equality of Numbers", in Logic, Logic and Logic, pp. 202-19; Alan Weir, "Neo-Fregeanism: an Embarrassment of Riches", Notre Dame Journal of Formal Logic 44 (2003): 13-48. 
relegated to the status of an impossibility whereas the other enjoys a privileged epistemological and foundational role.

In response to both the Bad Company and Embarrassment of Riches objections, a number of criteria have been considered for attempting to separate out the acceptable abstraction principles from the unacceptable ones, including conservativeness, permutation-invariance, non-inflation, modesty, non-paradox-exploitativeness, stability, irenicity, and others. ${ }^{2 \mathrm{I}}$ We need not review the technical details of these proposals here. Suffice it to say that it is generally agreed that no independently motivated (nonad hoc) criterion yet suggested unproblematically validates all those abstractions the neo-logicist wants (including not just Hume's principle, but those needed for real analysis, set theory and so on) and none that are incompatible with what they want. The search continues, and it is not yet hopeless, but even those proposals that come close to doing the work the neo-logicist requires them to do are complicated enough that it takes quite a bit of sophisticated logical and mathematical apparatus in the meta-theory to establish that a given abstraction principle has the requisite features. Presumably in order for such an abstraction to play the foundational epistemological and justificatory role the neo-logicist needs it to, the neo-logicist would not insist that someone must know that the principle in question is one of the acceptable ones (or even what it is to be an acceptable one), only that it in fact be an acceptable one. A comparison might be made to reliabilists about empirical knowledge, who do not typically require that someone know that her belief was reliably formed in order for it to be justified; it is enough that it was. In the $a$ priori realm, however, this seems a little more puzzling, and one cannot help but feel the strain wearing on the current Abstractionist doctrine.

\subsection{The Julius Caesar Problem}

This objection stems from Frege's own discussion of abstraction principles. Concerning the abstraction to the effect that the direction of line $a$ is identical with the direction of line $b$ if and only if they are parallel, Frege writes:

In the proposition:

${ }^{21}$ See, e.g., Kit Fine, The Limits of Abstraction (Oxford: Clarendon P., 2002); Wright, "Is Hume's Principle Analytic?", in Reason's Proper Study, pp. 307-32; Weir, "NeoFregeanism: an Embarrassment of Riches". 


\section{"the direction of $a$ is identical with the direction of $b$ "}

the direction of $a$ plays the part of an object, and our definition affords us a means of recognizing this object as the same again, in case it should happen to crop up in some other guise, say, as the direction of line $b$. But this means does not provide for all cases. It will not, for instance, decide for us whether England is the same as the direction of the Earth's axis-if I may be forgiven an example which looks nonsensical. Naturally no one is going to confuse England with the direction of the Earth's axis; but that is no thanks to our definition of direction. $^{22}$

Hume's principle, so far from providing the sort of definition of expressions for numbers that would allow us to understand the truth conditions of any sentences in which they appear in terms of already understood vocabulary, does not even go so far as providing the truth conditions for all identity statements for numbers. It only gives us the truth conditions for an identity claim formed with two names of numbers both explicitly describing the number as the number of some concept. It does not allow us to determine under what conditions the number of some concept would be identical to an object introduced another way, say England, or Julius Caesar. Hence, it does not really fix what expressions for numbers may or may not refer to, and hence does not really fix their meaning at all, or so one might argue.

Wright and Hale are of course well aware of this difficulty, and do have a response. ${ }^{23}$ To simplify: (HP) introduces the sortal concept of numbers, it tells us what sort of objects numbers must be, namely, those whose identity conditions are governed by whether or not concepts are equinumerous. Countries, like England, and people, such as Caesar, fall under other sortal concepts. It is of course notoriously difficult to spell out exactly what personal identity amounts to, but we can only assume that the question of whether or not something is identical to the person Caesar is legitimate only if there are determinate conditions under which one person is identical to another. According to Wright and Hale, an identity statement formed between entities falling under different sortals can only ever be true if the sortal of one is a sub-concept of the sortal of the other, and hence if the identity conditions of the one are a special

${ }^{22}$ Frege, The Foundations of Arithmetic, $\$ 66$.

${ }^{23}$ See Wright, Frege's Conception of Numbers as Objects, pp. II6-I7, and Hale and Wright, “To Bury Caesar ...”, in Reason's Proper Study, pp. 335-96. 
case of the identity conditions of the other. The sortal concept person could only be a sub-concept of the sortal concept number if, somehow, something's being identical to a given person could be explicated in terms of two concepts being equinumerous. Since this is evidently not how personal identity is to be construed, numbers are never people. This response still requires quite a bit of explanation to be perfectly clear, and many remain sceptical.

\subsection{Worries about an overfull ontology}

Objections of this sort come in various stripes. The first derives from simply objecting to the neo-logicists' allowing themselves to adopt a principle such as (HP) which is only satisfiable in infinite domains. ${ }^{24}$ The crude form of the objection leaves it at that: a logical truth is supposed to be-many would say, is defined as being — true in all models. ${ }^{25}$ But (HP) cannot be true for models with finite domains, and hence, one might argue, (HP) cannot have the status of a logical truth. This is a species of a more general line of criticism which argues, basically, that since logic itself must not make any substantive assumptions, and mathematics does by postulating the existence of various kinds of things, from numbers to functions to sets to what-have-you, nothing that entails the substantive existential claims of mathematics can be a part of logic. Logic must be devoid of ontological commitment, and should not even entail the existence of one object, much less infinitely many.

The crude form of the objection seems to be entirely question-begging. Why can't it be a logical truth that certain objects exist, so long as those objects are logical objects? Why must logic not have its own ontology? Other than by appeal to model theory, I've scarcely heard of a theory-neutral argument otherwise, and the attitude seems little more than a lingering by-product of the logical positivist insistence that analytical truths, as not empirically verifiable, must be completely devoid of factual content or significance-one that has inexplicably managed to remain despite the nearly universal rejection of most other positivist doctrines.

The model-theoretic argument, as stated, is not much better. A

${ }^{24}$ See Dummett, "Neo-Fregeans: in Bad Company"; Boolos, "Is Hume's Principle Analytic?"; and Rayo, "Logicism Reconsidered".

${ }^{25}$ I abstain from attributing this crude objection to any particular commentator on neo-logicism, but it is something I've heard in conversation. 
model-theoretic argument can only be used to establish that something isn't a logical truth once it is already established what should count as a legitimate model, and that cannot be established prior to considering what should count as a logical possibility and what should not. ${ }^{26}$ To my knowledge, no one objects to taking the first-order law of self-identity " $\forall x x=x$ " as a logical truth because it is not satisfied by models in which the identity relation would have a different extension. Those socalled models are simply excluded from consideration. Similarly, it is no objection to (HP) that it would come out as false if the biconditional double-arrow in the middle of it were reinterpreted as exclusive disjunction. As Wright insists, he takes the individual quantifiers as unrestricted, ranging over all the objects there are. If he is right in thinking that completely analytical arguments can convince us of the existence of infinitely many objects, there is no need to consider so-called models with finite domains as relevant.

Here one must further remember that the neo-logicist proof of an infinity of objects stems from (HP), by means of the bootstrapping described earlier. Demanding that they give some independent grounds for an infinity of objects before they are allowed to posit (HP) is just to dismiss their project before it starts, merely in virtue of where it ends. ${ }^{27}$

A more sophisticated version of this criticism, however, derives from scrutinizing the means whereby the neo-logicist argues for the existence of objects. The initial worry might be put this way. The neo-logicist argument for the existence of various logical objects, whether they be numbers or some other sort of abstraction, requires reconceptualizing an instance of the right half of some abstraction principle (AP) into an instance of the left half. While the right half is not existentially loaded and is not about any individual objects, the same cannot be said about the left half. A true instance of the left half provides a means of recognizing the existence of one object, and a false instance the existence of two distinct objects. How can one statement, which refers to objects and allows us to infer their existence, be a recarving of the content of another

${ }^{26}$ And this is even if we grant that the model-theoretic conception of logical truth and the logical consequence relation it defines get things right, which is itself questionable; see John Etchemendy, The Concept of Logical Consequence (Cambridge, Mass: Harvard U. P., 1990).

${ }^{27}$ Wright's explicit reply to such worries is similar; see, e.g., "Response to Dummett", in Reason's Proper Study, pp. 256-7I. 
that does not? One cannot introduce new objects into existence by stipulation or fiat. In response, the neo-logicist must appeal to a metaontological doctrine such as the priority of truth to reference according to which whether or not a certain claim refers to an object, and hence is committed to its existence, is not independent of the syntactic character of the expressions it uses. The commitment and reference to objects found on the left half of (HP) is a feature of its syntactic articulation; it refers to objects because it employs singular expressions for them.

Full evaluation of this response would more or less require settling almost all the big issues in ontology, meta-ontology, and philosophical semantics-you name it - and we cannot do this here. But it is worth probing a bit further into the ontological and meta-ontological commitments the priority thesis entails. In a recent paper, Matti Eklund has argued that in basing their position on this kind of priority thesis, neologicists commit themselves to a "radically promiscuous ontology", ${ }^{28}$ and in particular a kind of maximalism about existence. Any theory that can be evaluated as true "by ordinary standards" that intelligibly uses individual terms to refer to instances of some sortal concept $F$ will be successful in referring to $F_{\mathrm{s}}$; if we can intelligibly talk in terms of " $F_{\mathrm{s}}$ ", $F_{\mathrm{s}}$ exist. Cars must exist, not just molecules, since it is intelligible to speak about "the car in my garage" rather than the molecules in my garage arranged carwise. To use one of Eklund's examples, incars (that is, "would-be objects almost like cars except for the difference that they only exist when or insofar as they are inside garages"29) must exist, since sentences about "my incar" can be evaluated as true by ordinary standards if I explain what I mean. Objects like disjunctions and conjunctions must exist, since saying "The disjunction of $p$ and $q$ is entailed by the conjunction of $p$ and $q$ ", is a perfectly intelligible and, by ordinary standards, truthful restatement of “ $(p \wedge q) \vDash(p \vee q)$ ".

In their response to Eklund and others, ${ }^{30}$ Hale and Wright deny that they are committed to anything as inflationary as this kind of maximalism. However, they admit that they are committed to a view of the ontological status of the entities postulated by abstraction principles according to which there can be no serious doubts about the truth-preservation of

${ }^{28}$ Matti Eklund, "Neo-Fregean Ontology”, Philosophical Perspectives 20 (2006): 102.

${ }^{29}$ Ibid.

${ }^{30}$ Hale and Wright, "The Metaontology of Abstraction”, in Metametaphysics, ed. D. Chalmers (Oxford: Oxford U. P., 2009), pp. 178-212. 
the move from the right side of an instance of (AP) to the left. To secure this, these entities must be understood as less like concrete objects, or even "sparse" properties in a conservative metaphysics, but rather more like the abundant properties of a generous metaphysics that postulates them to exist whenever exemplification conditions can be specified for them. This is more or less to suggest that so long as there is a conceptual apparatus that allows for talk about such entities to be meaningful, or have a "sense" in Fregean terminology, reference is thereby guaranteed. Even if this does not lead to maximalism, it still leads to a fairly large ontology, merely on the basis of what can be meaningfully spoken of or conceptualized. Hale and Wright also admit that their countenancing of different "recarvings" of the same content make their view likely incompatible with what they call a "Tractarian" account of contents as states of affairs with a fixed form or structure uniquely mirrored by the syntactic form of sentences that express them.

\section{RUSSELL'S METHODOLOGY}

We are ready to turn to Russell, and Russell's confrontation with a number of issues which, if not identical to those discussed by contemporary neo-logicists, are at least highly related.

The first point of comparison I want to explore has to do with how the logicist project is to be conceived. Putting it that way is already to say something somewhat controversial, as there seems to be some disagreement about whether Russell himself was at all engaged in a logicist project as we now conceive of it. I have heard it claimed that Russell was not so much interested in showing the truths of mathematics to be truths of pure logic, but only two related projects: (I) showing that the vocabulary of pure (unapplied) mathematics can be reduced to logical vocabulary, and (2) identifying what the fundamental principles are from which the rest of mathematics can be derived, without necessarily requiring that such principles themselves be logically or analytically true. This reading is not without its merits, but I believe it is mistaken.

I believe Wright and others of like mind fundamentally conceive of their project as an epistemological one: to secure the epistemological footing of our beliefs in arithmetical truths by showing them to be entailed by truths of logic along with additional principles having roughly the epistemological status of definitions. This is certainly not how Russell conceived his project. If anything, Russell thought that mathematics was 
already on solid ground, epistemologically. Russell understood his project instead as an application of a general philosophical methodology that he was fairly explicit about in more than a dozen places. Indeed, it is somewhat of a mystery why this aspect of Russell's philosophy is not better known. ${ }^{31}$

The methodology consists of a two-phase process. In the first phase, one begins with a certain theory, doctrine or collection of beliefs, which is thought to be more or less correct, but is taken to be in certain regards vague, imprecise, disunified, overly complex or in some other way puzzling. The aim is to work backwards from these beliefs, taken as a kind of "data", to a certain minimal stock of undefined concepts and general principles which might be thought to underlie the original body of knowledge. The second phase consists in rebuilding or reconstructing the original body of knowledge in terms of the results of the first phase. More specifically, one defines those elements of the original doctrine deemed worth preserving in terms of the "minimum vocabulary" identified in the first phase, and derives or deduces the main tenets of the original theory from the basic principles or general truths so identified.

Russell calls this procedure "analysis", ${ }^{2}$ but it should be clear that it is not a process whereby we attempt to discover "what we meant all along" by a given collection of statements. The goal is rather to provide a replacement for the original doctrine, something that preserves what was desirable about the original, but taking a new form in which connections between various concepts are made clear, the logical interrelations between various theses of the theory are explicit, and vague or unclear aspects of the original terminology are eliminated. When successful, the concepts or assumptions giving rise to paradoxes, conundrums or other problems within the original theory are found to be wholly unnecessary

${ }^{3 \mathrm{I}}$ For some of Russell's explicit statements, see, e.g., PoM, pp. I-2, I29-30; PM I: 59; $O K E W_{4}$, pp. I44-5; IMP, pp. I-2; "The Philosophical Importance of Mathematical Logic", in Papers 6: 33; PLA, Papers 8: 157-63, 234-35; "Logical Atomism", in Papers 9: I63-8, I76-7; MPD, pp. 98-9, I62-3; "Reply to Criticisms", in Schilpp, p. 687 (Papers II: 22-3); and "The Regressive Method of Discovering the Premises of Mathematics", in Essays in Analysis (hereafter EA), ed. Douglas Lackey (New York: George Braziller, 1973), pp. 272-3. For discussion see Paul Hager, "Russell's Method of Analysis", in The Cambridge Companion to Bertrand Russell, ed. N. Griffin (Cambridge: Cambridge U. P., 2003), pp. 310-3I.

32 Though sometimes he reserves that term for the first phase only, dubbing the second the "synthetic" stage. 
or capable of being supplanted by something less problematic. Finally, the procedure arranges its results as a deductive system, and hence invites and facilitates the discovery of new results.

In providing an "analysis" of mathematics in terms of logic, Russell's aim was not to tell us what people mean when they speak of numbers, nor even what mathematicians mean. Preserving the vocabulary or syntactic structure of either ordinary statements about numbers or even the more sophisticated vocabulary of working mathematicians would have been completely unimportant to Russell, provided that which is useful and revealing about mathematical theories was retained.

As Russell described it, the process is akin to the procedure of hypothesis testing in the empirical sciences. The data to be explained by the resulting theory are the irreproachable aspects of the original body of knowledge, and they are thought to provide inductive evidence for the new theory. As Russell put things in Principia Mathematica:

The reason for accepting an axiom, as for accepting any other proposition, is always largely inductive, namely that many propositions which are nearly indubitable can be deduced from it, and that no equally plausible way is known by which these propositions could be true if the axiom were false, and nothing which is probably false can be deduced from it.

(PM I: 59)

Russell is explicit that in providing a logical foundation for mathematics, his goal is not to shore up the epistemological status of arithmetical claims such as " $2+2=4$ ". Indeed, those have to be taken as already more or less indubitable for the process to get underway.

If Russell's goal was not epistemological, can it be considered metaphysical? I believe so. The presupposition seems to be that insofar as the original body of knowledge was vague, and logical connections between the principles or concepts it assumes disguised, there was bound to be a disconnect between the form it took and the form of the facts to which it obliquely relates. Russell's metaphysics is "Tractarian", ${ }^{33}$ and thus unlike Hale and Wright's, in the sense that he thought that facts had definite logical structures independent of our conceptual schemes. Unlike Wittgenstein, ${ }^{34}$ however, Russell did not think that ordinary language's

${ }^{33}$ Or, more exactly, Wittgenstein's metaphysics in the Tractatus was (broadly) Russellian.

34 See Ludwig Wittgenstein, Tractatus Logico-Philosophicus (London: Kegan Paul, 
structure already reflected the structure of objective reality; indeed, the goal of analysis was in part to bring the structure of our scientific languages closer to that of reality.

\section{MATHEMATICAL TERMS AS INCOMPLETE SYMBOLS}

In applying the methodology just described to mathematics, Russell came to the conclusion that the minimum vocabulary and conceptual apparatus needed to reconstruct mathematics was that of logic. It is difficult to say, given their rather different conceptions of their projects, whether or not this is a point of agreement with the contemporary neologicists. Of course, while Wright does not think that mathematical vocabulary can be defined outright in terms of logical vocabulary, he does think that anyone armed with an understanding of the language of second-order logic is in a position, in principle, to gain an understanding of mathematical vocabulary and concepts by means of relating the latter to the former. ${ }^{35}$

Russell had been exposed early on to the notion of a "definition by abstraction" in the work of Peano and his associates, who used what they called the "principle of abstraction" liberally. ${ }^{36}$ (Indeed, the Abstractionists Hale and Wright would less misleadingly be labeled "neo-Peanists" rather than "neo-Fregeans".) As Russell stated it, this principle asserted that "when there is any relation which is transitive, symmetrical and (within its field) reflexive, then, if this relation holds between $u$ and $v$, we define a new entity $\phi(u)$, which is to be identical with $\phi(v)$ " (PoM, \$2IO). The $\phi$ here is in effect a function; it is the same as the $\Sigma$ in (AP) above. Russell regarded functions as derived from relations, and so took the principle to postulate a relation $S$ for every equivalence relation $E$ such that $u$ would bear $S$ to the same object to which $v$ bears $S$ just in case $u$ and $v$ are related by $E$. Russell realized, however, that there could be many such $S$ s - which is just the same insight as that in (AP), there may be many different interpretations of $\Sigma$ that would make it true. In effect, this is to realize a version of the Julius Caesar problem: satisfying

Trench, Trubner, 1922), \$5.5563.

35 See Wright, "On the Harmless Impredicativity of $\mathrm{N}^{=}$, , in Reason's Proper Study, pp. 229-55.

${ }^{36}$ See, e.g., Giuseppe Peano, Formulaire de mathématiques, Vol. I (Turin: Bocca, I895), p. 45. 
(AP) for a given equivalence relation $E$ is not enough to pin down what $\Sigma(\kappa)$ and $\Sigma(\tau)$ must be. ${ }^{37}$ In his earliest work in formal logic (Papers 3: 320), Russell sought to overcome this by taking $S$ to be the relation holding between $u$ and the class of objects to which it bears $E$, thereby giving a specific reference to " $\phi(u)$ " (or " $\Sigma(\kappa)$ " if you prefer). With $\phi(u)$ taken as the class of all $x$ to which $u$ bears $E$, Russell, early on, regarded the principle of abstraction as "capable of proof" (PoM, \$157) from the basic principles of class theory.

In his mature work, of course, Russell came to have doubts about the reality of classes, eventually coming to the conclusion that class terms are "incomplete symbols", or symbols which do not stand directly for entities, but nevertheless contribute to the meaning of expressions in which they appear in a more complicated way. There is a sense, then, in which the mature Russell too believed that mathematical vocabulary could not be defined outright in logical vocabulary. Almost without exception, any mathematical term, for a number, for a function, for a mathematical relation, after Russell's analysis, becomes for Russell the kind of expression for which one cannot find a single unified expression formed using logical vocabulary that could replace it. Rather, there is a method whereby any sentence in which that term appears can be translated, whole-scale, into a sentence containing no non-logical constants. ${ }^{38}$

Let us take as our example the closest notation found in Principia Mathematica to the neo-logicist \#( ) involved in (HP), which Russell writes "Nc". In appearance, this was used as a functor applied to a class term $\alpha$. Modernizing Russell's notation somewhat, and applying (or simplifying) the results of a number of intermediate definitions, we have:

$$
\mathrm{Nc}(\alpha)={ }_{\mathrm{df}} \hat{\beta}(\beta \cong \alpha)
$$

The number of class $\alpha$ is the class of all classes equinumerous with $\alpha$. Of course, this is not the end of the analysis, as according to Russell's "no class" theory, terms for classes may be eliminated contextually. In the

37 See also Russell, "On Some Difficulties in the Theory of Transfinite Numbers and Order-Types”, in EA, pp. I49-5I.

${ }^{38} \mathrm{Or}$, more precisely, containing no non-logical constants as part of the contribution it makes to the sentence. The analysis of a mixed sentence such as "I have two brothers," will contain non-logical constants (representing me, the brotherhood relation, etc.), but not any that derive from the word "two". 
case of a term for a class of individuals, i.e., one of the form $\hat{z}(\psi z)$, we have, by $P M$ 's $* 20.0$ I:

$$
\phi[\hat{z}(\psi z)]=_{\mathrm{df}} \exists F[\forall x(F ! x \leftrightarrow \psi x) \wedge \phi(F ! \hat{z})]
$$

Quantification over classes, using dummy Greek variables, is to be eliminated in terms of quantification over propositional functions defining classes, so that:

$$
\forall \alpha \psi \alpha={ }_{\mathrm{df}} \forall F \psi[\hat{z}(F ! z)]
$$

Such quantification over classes is not identical to, but is isomorphic with, quantification over individuals, so that for each quantifier rule for genuine individual variables, there is a corresponding one for class variables, and vice versa (PM г: 196).

In the case of classes of classes, such as $\mathrm{Nc}(\alpha)$, a statement "about" one may be analyzed as involving quantification using an even higher-type propositional function variable along with the kind of quantification over classes just defined. By $P M$ 's $* 20.08^{39}$ :

$$
\Phi[\hat{\beta}(\psi \beta)]=_{\mathrm{df}} \exists M[\forall \alpha(\psi \alpha \leftrightarrow M ! \alpha) \wedge \Phi(M ! \hat{\beta})]
$$

These contextual definitions, along with certain conventions for resolving potential scope ambiguities, should in principle allow us to eliminate the expression " $\mathrm{Nc}(\alpha)$ " in any context in which it appears, so that the result becomes a statement involving higher-order quantification, containing no constants besides logical constants.

The contextual definitions for class terms along with the standard higher-order definition of identity gives identity statements formed using the class term incomplete symbols their usual extensionality, i.e.:

$$
\hat{\beta}(\psi \beta)=\hat{\beta}(\chi \beta) \leftrightarrow \forall \beta(\psi \beta \leftrightarrow \chi \beta)
$$

39 PM's contextual definition of classes does not directly allow for a theory of "multisets", but multisets can be defined using PM's class logic by means of relations between sets, e.g., sets of ordinals, and the would-be members of the multiset, with the "multiplicity" of a given member of the multiset defined as the number of terms of the original class related to it. 
And so, finally, in the end, we get the following versions of Hume's principle, not as basic assumptions of the system, but as consequences of the reconstructed class theory and the definition of numbers utilized therein, along with the purely logical result that equinumerosity is an equivalence relation. We have:

$$
\forall \alpha \forall \beta(\mathrm{Nc}(\alpha)=\mathrm{Nc}(\beta) \leftrightarrow \alpha \cong \beta)
$$

And indeed, given how quantification over classes is to be construed, this can be rewritten:

$$
\left(\mathrm{HP}^{\mathrm{R} 2}\right) \quad \forall F \forall G(\mathrm{Nc}(\hat{z}(F ! z))=\operatorname{Nc}(\hat{z}(G ! z)) \leftrightarrow F ! \hat{x} \cong G ! \hat{x})
$$

If we were to abbreviate " $\mathrm{Nc}(\hat{z}(F ! z))$ " as simply \#(F), we'd get something that appears almost identical to (HP) above.

In the end, Russell too believes that someone possessing an understanding and knowledge of higher-order logic has all that he or she needs in order to gain an understanding of the truth-conditions of mathematical statements as he analyzes them. However, this is not done by simply stipulating that there are terms referring to objects whose identity conditions are to be governed by (HP), but rather by inventing a new way of writing statements of pure logic so that they appear to utilize terms for objects having such identity conditions, but in which it can be seen, when the contextual definitions are unpacked, that having these identity conditions is simply a by-product of the definitions.

One of the chief differences, of course, is that an expression of the form " $\mathrm{Nc}(\alpha)$ " is not understood by Russell as a genuine term. Russell, unlike Wright, does not take surface grammar as indicative of referential power. Russell rejects the priority principle. In a sense, Russell admits that certain statements expressible using logical vocabulary alone can be "reconceived" as (or really made definitionally equivalent to) statements with apparent terms for numbers. This is not enough to secure the result that these apparent terms are terms, properly speaking, or do have their own referential powers. All connection with entities comes in indirectly through the quantificational apparatus of the system. A term for a number is in Russell's parlance an "incomplete symbol", meaning that it contributes in a regular way to any sentence in which it appears, but is not meaningful by naming or representing a singular entity on its own; it requires a context of use so that it can be resolved into a statement of 
greater complexity.

Russell uses the phrases "logical constructions" and "logical fictions" as labels for those apparent "things", such as classes and numbers, which certain discourse appears to be about only because of the use of incomplete symbols that are seemingly for them within sentences with welldefined truth conditions in the sense just described. Russell's mature version of the "principle of abstraction", as he sees it, allows us to do the work of the kind of abstraction principles found in the Peanists, or in the work of Hale and Wright, but without postulating any new entities. Hence, the mature Russell claims that it might just as well be called "the principle which dispenses with abstraction" (OKEW, p. 40; Papers 9: I65-6). This principle itself is not taken as basic, nor are the "apparent" entities that it seems to point to.

\section{ADVANTAGES OF INCOMPLETE SYMBOLS}

Full assessment of Russell's views on the nature of incomplete symbols and logical constructions, and a relative assessment of them as a metaontology rivalling the Abstractionist neo-logicist position is not possible in the present context; it leads straightaway to many of the fundamental questions in philosophical semantics and metaphysics. However, they do possess a number of advantages when it comes to the logicist project in the philosophy of mathematics in particular. Those advantages are these:

(I) It provides direct insight into how it is that numbers can be introduced as entities whose truth conditions are governed by the obtaining or non-obtaining of an equinumerosity relation. This fact about numbers is neither something that needs to be assumed as a "new fundamental principle" nor the result of a definition which is not really a definition. The way in which numbers are introduced as logical constructions guarantees, purely logically, that they are so individuated. As Russell put it later, "none of the raw material of the world [i.e., the genuine entities it comprises] has smooth logical properties ... whatever appears to have such properties is constructed artificially in order to have them" (PoM, 2nd edn., p. xi). Numbers are constructed in order to obey Hume's principle.

(2) It provides an immediate solution to the Julius Caesar problem. The contextual definitions offered by Russell $d o$ provide a means for eliminating number terms in any context in which they may appear. Of course, the precise means of eliminating them puts constraints on what 
contexts they may meaningfully appear in. The identity sign is a defined sign in Russell's higher-order logic, and because of the definition, identity statements are only meaningful when the two sides are flanked by expressions of the same logical type. Number terms are construed as classes of classes, and such class terms are eliminated in favor of quantification over higher-order propositional functions. The consequence is that number expressions may not be meaningfully placed in a position where a name of an individual may go. Hence, the question of whether or not Julius Caesar is 0 is not a meaningful question. It is no skin off Russell's nose that ordinary language treats these two expressions as syntactically similar, and hence, can pose the question. Russell's analysis of mathematics is offered as a replacement for the mathematical discourse of everyday life and even ordinary mathematics. Indeed, one of the purposes of the methodology is to eliminate conundrums created by the more haphazardly organized conceptual system with which we began.

(3) Rather more indirectly, it provides solutions to both the Bad Company and Embarrassment of Riches problems.

We have seem how it is that a version of (HP) can be obtained, not as a new fundamental assumption, but as a derived theorem from the underlying logical laws as well as the precise nature of the construction of numbers. Something similar can be done for any abstraction principle that might be offered by the neo-logicist. The general form is again:

$$
\forall \kappa \forall \tau(\Sigma(\kappa)=\Sigma(\tau) \leftrightarrow E(\kappa, \tau))
$$

One need only define $\Sigma(\kappa)$ as $\hat{\mu} E(\kappa, \mu)$, where $\mu$ is another variable of the appropriate type, and then, provided that $E$ is indeed an equivalence relation, a result of the shape above is obtainable. Indeed, reconstructing discourse about would-be entities with such identity conditions by means of this method is something Russell repeatedly and self-consciously advocated in works both early and late. ${ }^{40}$

Indeed, we have already seen a higher-type version of Basic Law $\mathrm{V}$ above in $\left(\mathrm{Ext}^{\mathrm{R} *}\right)$, a version for classes of individuals, closer to Frege's version, is also obtainable:

$\left(\mathrm{Ext}^{\mathrm{R}}\right) \quad \forall F \forall G(\hat{x}(F ! x)=\hat{z}(G ! z) \leftrightarrow \forall x(F ! x \leftrightarrow G ! x))$

${ }^{40}$ See e.g., PLA, Lect. viri; "Logical Atomism", Papers 9: I64-5. 
Order-types, or what Russell called "relation numbers", of which ordinal numbers are a species, were obtained in Principia by this method, yielding a result of the shape of (Ord) above.

However, unlike their Abstractionist counterparts, these Russellian versions do not lead to contradiction. The reason is that the expressions flanking the identity sign on the left half of the principle are no longer regarded as genuine terms, and hence do not name individuals. Instead, as we have noted, these are incomplete symbols that can only be defined away in context.

Given the nature of the construction of class terms, the type theory of Principia requires " $\hat{\mu} E(\kappa, \mu)$ " to have a distinct logical type from symbols of the type of " $\kappa$ " or in terms of which " $\kappa$ " may be constructed. The Russellian principle $\left(\mathrm{Ext}^{\mathrm{R}}\right)$ does not introduce new individuals which may or may not be included in the very extensions that $\left(\mathrm{Ext}^{\mathrm{R}}\right)$ deals with, and hence does not lead to Russell's paradox. The Russellian version of (Ord) does not give us new entities that can fall in the field of the relations whose relation types are dealt with by (Ord) itself. This prevents it from leading to the Burali-Forti paradox. So there is no bad company here.

The Embarrassment of Riches problem is solved in almost precisely the same way. The riches are no longer embarrassing. One may, if one wishes, introduce an incomplete symbol for "the parity of $F$ ", defined as the class of all classes that are extensions of concepts differing evenly from $F$, or something similar, thereby arrive at something of the form of (PP). This version, however, is not incompatible with the Russellian version of Hume's Principle. The inconsistency between them is a result of the proof of an infinite domain of objects from (HP), and a similar result leading to the consequence that the domain must be finite in the case of (PP). The way in which (HP) leads to an infinity of entities - the bootstrapping described earlier-is blocked if the number expressions flanking the identity on the left are not taken as genuine terms for objects. Without (HP) delivering new objects which can in turn be used to define ever more expansive concepts applicable to such objects, themselves in turn to be fed back into the right side, the unending expansion of the known domain of quantification does not get underway. The derivation of a finite domain from the parity principle proceeds in a similar way. When the identity statements on the left involve logical constructions, the principles superficially similar to the neo-logicist's abstraction principles play nicely with one another. Indeed, their mutual consistency 
can be proven. ${ }^{4 \mathrm{I}}$

(4) And for reasons that should already be clear, one need have no worries that the Russellian approach leads to a "radically promiscuous" ontology, as, indeed, it entails no genuine ontological commitments beyond those involved in the basic axioms of higher-order logic and higher-order quantification. The incomplete symbols that can be used to emulate talk about numbers or functions or relations or classes really introduce a new way of talking; the logical constructions they introduce are not "new things" in addition to what one is committed to by means of higher-order logic alone. Any appearance one may have that one is defining into existence any new entities is an illusion engendered by taking the surface structure of the defined symbols uncritically and without properly understanding the contextual definitions. The same cannot uncontroversially be said about understanding the neo-logicist versions of abstraction principles as implicit definitions.

Indeed, it seems that the Russellian approach provides a much more intuitive picture of how it is that a state of affairs can be "reconceptualized". The reality behind the truth that there are equally many planets in our solar system as there are members of the Brady family is not one with a plasticene nature that can be molded to be seen as an identity between numbers on the one hand, and an existential claim about a I-I correspondence holding between two concepts on the other. Instead, we have a single truth which, when fully articulated, has an univocal structure, although it is within our means to invent modes of symbolism that hide, or (perhaps better) encode or encrypt, aspects of the structure with a differently appearing surface form.

\section{THE LOSS OF INFINITY}

None of this is to say that the conception of mathematical terms as incomplete symbols, and hence mathematical objects as logical constructions, is without its costs. Indeed, the costs are the flip side of the coins for the advantages. The Russellian version of Hume's principle cannot

${ }^{4}$ I The proof would be remarkably similar to the proof given by Weir that all predicative abstraction principles are consistent with one another; see Weir, "Neo-Fregeanism: an Embarrassment of Riches". Of course, their mutual consistency with one another is also a corollary of the general consistency of the logic of $P M$, which can be shown relative to set theories as weak as Z. 
be used to establish the existence of an infinity of objects. If the number of objects is $n$, the number of classes of objects is $2^{n}$, and the number of classes of classes of objects is $2^{2^{n}}$. Hence if $n$ is finite, so is the number of classes of classes, and therefore, the number of numbers. Since numbers are not themselves objects on this approach, bootstrapping is out. Standard Peano arithmetic entails the existence of infinitely many distinct numbers, and hence, cannot be obtained, unmodified, from the Russellian version of Hume's principle. In order to attain standard Peano arithmetic outright in Russell's system, one needs to assume that the domain of quantification for the individual variables is infinite, usually taken in the form of assuming that the universal class of individuals does not have a natural number as its cardinality.

Russell was explicit about this in many places and, in works such as Introduction to Mathematical Philosophy, admitted that logic alone is powerless to convince us of the existence of an infinity of individuals. Indeed, Russell is often read here as simply giving up the game, admitting that at best the vocabulary of mathematics reduces to that of logic, not the foundational truths. ${ }^{42}$ Together with the multiplicative axiom (Russell's version of the axiom of choice) and the axiom of reducibility, the assumption of an infinity is seen as an evidently non-logical assumption that disqualifies Russell's foundational project as any kind of success in establishing logicism in a strong sense.

These criticisms seem too quick to me, and don't do proper justice to the nature of Russell's project as a reconstructive one. The issues they raise also don't get at the heart of the differences between Russell and the neo-logicists. Full examination of the multiplicative and reducibility axioms would require a more in-depth study than is possible here. I shall content myself with some rather snarky replies. For the multiplicative axiom, we need only mention here that it is not needed for the lower parts of Russell's reconstruction of mathematics, i.e., its treatment of the truths of natural number theory or real analysis. The areas in which it would be needed, e.g., the arithmetic of infinite numbers, are areas that neo-logicists have themselves only begun to explore, and it is not clear that their approach would fare any better without a similar notobviously-logical axiom. It does raise a number of issues regarding the

${ }^{42}$ See, e.g., William Kneale and Martha Kneale, The Development of Logic (Oxford: Oxford U. P., 1962), p. 699; Alan Musgrave, "Logicism Revisited", The British Journal for the Philosophy of Science 28 (1977): II2. 
proper interpretation of Russell's higher-order logic_- especially as taking the postulation of "choice sets" for infinite collections to be analyzable in terms of quantification over "propositional functions" seems to require there to be infinitely complex propositional functions; but these are issues best treated separately from the comparison of Russell's form of logicism and the more recent forms.

The axiom of reducibility is also, I think, not a fruitful place to look for help in assessing the relative merits of the Russellian versus the Abstractionist approaches to logicism. The need for it at all stems from Russell's adoption of a ramified theory of types, rather than a more straightforward simple theory of types. Wright, Hale et al. assume something closer to the latter in adopting an impredicative comprehension principle for the second-order logic in which they couch their theories. The disagreement here, I think, once again has to do with the proper understanding of the semantics and justification for higher-order logic generally, and while there are substantial issues there to be discussed, a wouldbe current day logicist would be free to adopt the neo-logicist conception of second-order logic, along with the Russellian logical constructionist account of mathematical discourse, which would render the issue of the axiom of reducibility completely moot.

The issues raised by the need to assume a principle of infinity rather than having a proof of it from one's basic logical assumptions seems a more suitable place to frame the debate. However, here is a little known fact: Principia Mathematica does not have an axiom of infinity. ${ }^{43}$ Instead, rather than proving those results of standard Peano arithmetic that require the assumption of an infinite domain, they prove instead a conditional where the assumption of an infinite domain of individuals is taken as the antecedent and the standard result as consequent. In particular, rather than proving the fourth Peano postulate, i.e., that no two distinct natural numbers have the same successor, they instead prove that if there are infinitely many individuals, then this is the case. This is similar to how the multiplicative axiom is treated, but different from how other "primitive propositions" are, including the reducibility axiom. Unlike reducibility, for example, Whitehead and Russell apparently do not believe that even their inductive or "regressive method" provides suffi-

${ }^{43}$ Although to be fair, the misconception that it is an axiom of $P M$ is Russell's own fault, since he himself describes it that way in various places ( $L K$, p. 97; IMP, p. I3I). 
cient justification for taking the infinity axiom to be true. Hence it is left as an undischarged antecedent or "hypothesis". ${ }^{44}$

This maneuver arguably opens up Russell's logicism to the charge of "if-thenism", ${ }^{45}$ that is, that it fails actually to show that mathematical truths are logical truths; it only shows that mathematical truths are consequents of logical truths. This, however, is trivial. Any truth $p$ whatever is the consequent of some logical truths (e.g., if $\mathrm{p}$ then $\mathrm{p}$ ), and even empirical sciences can often be arranged as deductive systems so that the conditional from the conjunction of their basic principles to the consequences of those principles would be logical truths. Should we then regard Russell's adoption of an infinity antecedent as trivializing his logicist ambitions?

It depends on whether, at the end of the day, you insist that the real pure mathematical truth is necessarily Peano postulate 4, as traditionally conceived without any suppressed antecedents. Again, we must remember that Russell's project was a reconstructive one; his aim was not to preserve all aspects of the original ways of conceiving and talking about mathematical so-called entities. If a different logical form better represents the form of the actual fact hinted at by the original doctrine, it is to be preferred. ${ }^{46}$

Indeed, even without buying into a reconstructivist approach like Russell's, thinking that the true mathematical principles that working mathematicians in fact prove have hidden assumptions that are not always made explicit, should not be at all controversial. No one would object to logicism on the grounds that one cannot prove the Pythagorean theorem in the simple form $a^{2}+b^{2}=c^{2}$ outright. Obviously, one can

44 Treating infinity in this way, different from reducibility, was apparently important enough to Russell that when Whitehead mistakenly left it off as a restriction to various results in the submitted draft of Volume II, Russell paid to have the mistake corrected; see I. Grattan-Guinness, The Search for Mathematical Roots, 1870-1940 (Princeton: Princeton U. P., 2000), pp. 385, 400.

45 See Hilary Putnam, "The Thesis that Mathematics Is Logic", in Bertrand Russell: Philosopher of the Century, ed. R. Schoenman (London: Allen \& Unwin, 1967), pp. 273-303; Musgrave, "Logicism Revisited"; Alberto Coffa, "Kant and Russell”, Synthese 46 (I98I): 247-63.

${ }^{46}$ For a longer discussion of the complex interplay between Russell's attitude towards various mathematical notions both pre-and post-analysis, as well as comparison of these attitudes with those of the Abstractionist school, and even a discussion of how these issues relate to the debate over if-thenism, see Sébastien Gandon, Russell's Unknown Logicism (New York: Palgrave Macmillan, 20I2). 
only prove that if $c$ is the length of the hypotenuse of a right triangle, $a$ and $b$ are the lengths of the other sides, and the triangle exists in a Euclidean space, then $a^{2}+b^{2}=c^{2}$. These assumptions are not part of what immediately comes to mind when one thinks of the theorem, and especially the part about the triangle existing in a Euclidean space is apt to be taken simply as a background assumption to the working mathematician. That there are any applications of the theorem to any actual triangles is not a part of what the mathematician is even attempting to prove.

It might be argued ${ }^{47}$ that pure mathematics ought not to take a stand on whether or not there are infinitely many individuals, or even, by extension, whether or not there are infinitely many numbers. Such issues involve the application of mathematics to the concrete world. Similarly, it is appropriate that Russell did not prove or even attempt to prove that the axioms of any given Euclidean or non-Euclidean geometry actually hold of space. It is a matter of the application of these various mathematical systems to the concrete world which of these sets of axioms actually describe space. If the world we actually live in has infinitely many individuals, then Peano arithmetic will have an application to it; if not, then not. If all that is needed for the application of mathematics are suitably large finite numbers, then, as Russell points out, the relevant results are already forthcoming, even without any new existential posits, at some stage of the hierarchy of types (IMP, p. I33).

\section{CONCLUSION: PROSPECTS FOR FUTURE LOGICISMS}

Obviously here, we have only begun to scratch the surface of the philosophical issues that get raised by Russell's form of logicism, or even the issue of infinity in particular. However, it seems clear that it would be very premature simply to conclude that this issue alone ruins the prospects for any kind of Russellian or neo-Russellian logicism. By comparison to the litany of problems that continue to plague Abstractionist-style neo-logicisms, this actually seems like a far less daunting route for a twenty-first century logicist to explore.

And indeed, there are some alternative avenues that might look

${ }^{47}$ And indeed, has been so argued—see Gregory Landini, Russell (London: Routledge, 2OII), Ch. 2. 
tempting. One such would be to obviate the need for adding a principle of infinity by seeking some new argument to the effect that an infinity of objects can be regarded as a logical or analytical truth. I argued earlier that I have heard no good argument to the effect that in principle logic cannot have its own ontological commitments. I am somewhat more sceptical about this option, however, since the most plausible routes would seem to involve moving away from a Russellian approach of treating things that have "smooth logical properties" as constructions rather than among the "raw material" of the world.

If taking an infinity of objects as a logical truth is not to be urged simply on inductive grounds alone ${ }^{48}$ — since as Russell puts it, it allows us to deduce many truths we are already convinced of, and no equally plausible way of explaining their truth is known - then our argument for an infinity must establish the existence of objects of a certain kind. In order to ensure that the number of these things is indeed infinite, we must be able to individuate them. If the objects in question are not classes, or sets, or numbers, or concepts-as-things, then what are they to be? The only other kind of thing I can think of would be intensional entities, be they propositions or something more like reified meanings, Fregean senses, or whatnot. Quine notoriously worried that these were "creatures of darkness", ${ }^{49}$ bewildering us with their elusive identity conditions. My own researches into the subject have only confirmed Quine's complaint, and, I think, Russell's reasons for eventually abandoning propositions as self-subsistent objective beings are of a piece with his attitudes towards classes and numbers. ${ }^{50}$

Indeed, it is striking that meanings have recently been proposed as additions to the list of things that might perhaps be best introduced by means of abstraction principles. ${ }^{5 \mathrm{I}}$ One would need very good reason to think that this road is not just a road to bad company if the neo-Russel-

${ }^{48}$ Although I think that view may have its defenders too; see, e.g., Matthew McKeon, "Logic and Existential Commitment", Logique et analyse 47 (2004): 409-23.

49 Quine, "Quantifiers and Propositional Attitudes", in The Ways of Paradox and Other Essays, rev. edn. (Cambridge, Mass: Harvard U. P., I975), p. I88.

50 For more on this point, see Klement, "Russell, His Paradoxes, and Cantor's Theorem", 2 parts, Philosophy Compass 5 (2010): 16-28 and 29-4I; an online journal (http://onlinelibrary.wiley.com), accessed January 2013.

${ }_{\text {5I }}$ For discussion, see, e.g., Peter Simons, "Gray's Elegy without Tears: Russell Simplified”, in On Denoting I905-2005, ed. G. Imaguire and B. Linsky (Munich: Philosophia Verlag, 2005), pp. I32-3. 
lian logicist is to maintain any advantage over the neo-Fregean Abstractionist.

However, this is certainly not conclusive evidence that prospects for defending a neo-Russellian logicism might not be fruitfully explored down this avenue either. I should also say that I do not think I have given any knock-down arguments to the effect that only neo-Russellian forms of logicism have any prospects in the twenty-first century. I have only aimed to argue that they have certain attractions, and avoid many of the more robust difficulties continuing to plague the dominant $\mathrm{Ab}$ stractionist forms of neo-logicism. I am by no means ready to make the same mistake that so many have made with regard to logicism as a whole: pronounce its death, or even the death of one of its forms, prematurely. 